Casos clínicos.

\title{
SINDROME DE KLIPPEL-TRENAUNAY
}

\author{
(Hemangiectasia hipertrófica de Parkes Weber).
}

\author{
Relato de un caso.
}

\begin{abstract}
Por el Prof. HERNAN HEVIA y tos Dies. JORGE ROSSELOT Y RODOLFO BURDACH
Clínica Universitaria do Piel y Sifilis dé: Prof. L. Prunés.

Hosp. M. Arraran. Catedras de Pediatria de los Profs. A. Bazza Goñi y Julio Mencghe'..o R.
\end{abstract}

Klippel y Trenaunay, en 1900, describieron con el título de naevus varicoso ósteo-hipertrófico un cuadro clínico caracterizado por la existencia de naevus del miembro inferior, várices del lado afectado e hipertrofia de los tejidos y esqueleto en el segmento comprometido. Los autores citados atribuyeron gran importancia al naevus, pensando que era ésta la lesión fundamental; sin embargo, con posterioridad se han descrito formas ósteo-hipertróficas sin naevus o variedades disociadas, en que se comprueban naevus en el lado opuesto a aquél en que aparecen várices e hipertrofia segmentaria. Ya en 1869, Trelat $y$ Monod, habian relatado observaciones similares a las señaladas por Klippel y Trenaunay; posteriormente se han señalado nuevos casos de esta afección, entre los que cabría referirse a los descritos por Apert, Danlos y Flandin, en 1909; Leroux y Raoul, en 1910; Babonneix y Lance, en 1924 y Apert, en 1931 (Servelle. 1945).

En 1918, Parkes Weber describió un cuadro clínico con anomalías semejantes a las que constituyen el sindrome de Klippel-Trenaunauy, bajo el título de "Hemangiectasia hipertrófica". En esta afección existirian angiomas, várices, aneurismas cirsoídeos o arterio-venosos e hipertrofia segmentaria (Aimes, 1950).
Pautrier y Ullmo, en 1928, describen los hallazgos histológicos advertidos en un paciente de 17 años de edad, con hemangiectasia hipertrófica. Señalan que las lesiones más importantes corresponden a hiperqueratosis, dilatación vascular junto a la epidermis, esclerosis del tejido conjuntivo e infiltración histiocitaria, con acúmulo de pigmento férrico en el dermis. Touraine (1936) señala que en ciertos casos aparecen junto a las várices, angiomas e hipertrofias regionales, lesiones noduiares o placas irregulares escamo-verrucosas e hiperqueratósicas.

Servelle, en 1945 , relata las observaciones de dos pacientes de 16 y 17 años, respectivamente, que presentaron várices, hipertrofia ósea regional y coexis. tencia de naevis en uno de los casos. Es importante señalar que la venografía en los dos enfermos comprobó anoma. lías vasculares (bridas o ausencia de la vena safena), que podrían ser el punto de partida de fenómenos de éxtasis con hipertensión venosa, responsable a su vez de las várices, angiomas y aumento de la longitud ósea. Apoya la interpretación patogénica de Servelle, el resultado eficaz que se ha logrado en la reducción del crecimiento óseo desmesurado, después de intervenciones quirúrgicas que suprimen la hipertensión ve- 
nosa, corrigiendo las anomalias vasculares.

En niños, Casaubon y Chait (1930), en Argentina $y$ Cienfuegos y Mattar (1931) entre nosotros, han relatado observaciones que presentaron coexistencia de angiomas e hipertrofias segmentarias, similares a las descritas por Parkes Weber.

En el curso del presente año hemos tenido oportunidad de observar en un lactante de 1 año y 6 meses de edad, lesiones angiomatosas, várices e hipertrofias regionales, con las caracteristicas propias del síndrome de KlippelTrenaunay y cuyos detalles clínicos e histológicos se anotan a continuación.

M. G. P. - Obs. N" $51 / 89418$.

Edad: 1 año y 6 meses.

Antecedentes familiares: Madre, de 21 años, sana, serología (-) en 1949. Padre, de 21 años, sin examen serológico. Dos hermanos, de 4 y 3 años. sanos. No hay contagio tubenculoso.

Antecedentes personales: Parto eutóclco, de término. Pesó al nacer $4 \mathrm{~kg}$. Perjodo del recién nacido normal. Allimentado al pecho durante 6 meses en forma exclusiva; destete en el noveno mes $y$ antes complemento de leche de vaca diluida en forma excesiva. Desde el noveno mes se administra leshe de vaca pura, $1 / 4$ litro diario y resto del régimen medianamente satisfactorio. No so han proforcionado vitaminas. El apetito ha stdo bueno. Sostuvo la cabeza a los 3 meses, se sienta el noveno mes, aun no anda. Dentición en el décimo mes. Habla desde el año.

Controlado en Unidad Sanitaria Qulnta Normal. Ha presentado episodios dispépticos y gripales.

Enfermedad actual: Desde el nacimiento se advirtió aumento notorio de volumen de las extremidades derechas en sus segmentos distales (mano y pie). Hay un aumento semejante, pero más discreto de las extremidades izquierdas. Se aprecló también la existencia en todo el cuerpo, a excepción de la cara, de manchas rosado-violáceas.

F,xamen físico: Peso: $10.300 \mathrm{~kg}$. Talla: 78 cm. Talla sentado: $43 \mathrm{~cm}$. C. craneana: 48 $\mathrm{cm}$. C. toráxica sup.: $51 \mathrm{~cm}$. C. toráxiea inf.: $50 \mathrm{~cm}$. T.: $36.5^{\circ}$. Pulso: 89. Resp.: 28*.

Lactante en buen estado nutritivo, permanece sentado en la cama, observando con atenclón lo que sucede a su alrededor. No se sostiene en pie

Piel: Se aprecian manchas de color rosadovioláceas, que están separadas por plel de tinte normal, confluentes en algunas zonas $y$ que interesan la mayor parte dal dorso, la pared abdominal, la extremidad superior izquierda y el muslo derecho. En otras zonas no hay tendencia confluente. Está respetado el segmento cefálico. En la base del hemitórax izquierdo, en la región dorsal y en la cara anterior se aprecian venas dilatadas en forma manifiesta. En las regiones supraesternal y clavicular izquierda existen trayectos venosos con menor grado de dilatación. Se observan también aumentos de volumen múltiples, con aspecto de cojinetes: blandos, con sensacićn de paniculo adiposo, a nivel del dorso, en la región pectoral $\mathrm{y}$ en las 4 extremidades, especialmente en las derechas (Foto $\mathbf{N}^{\prime}$ )

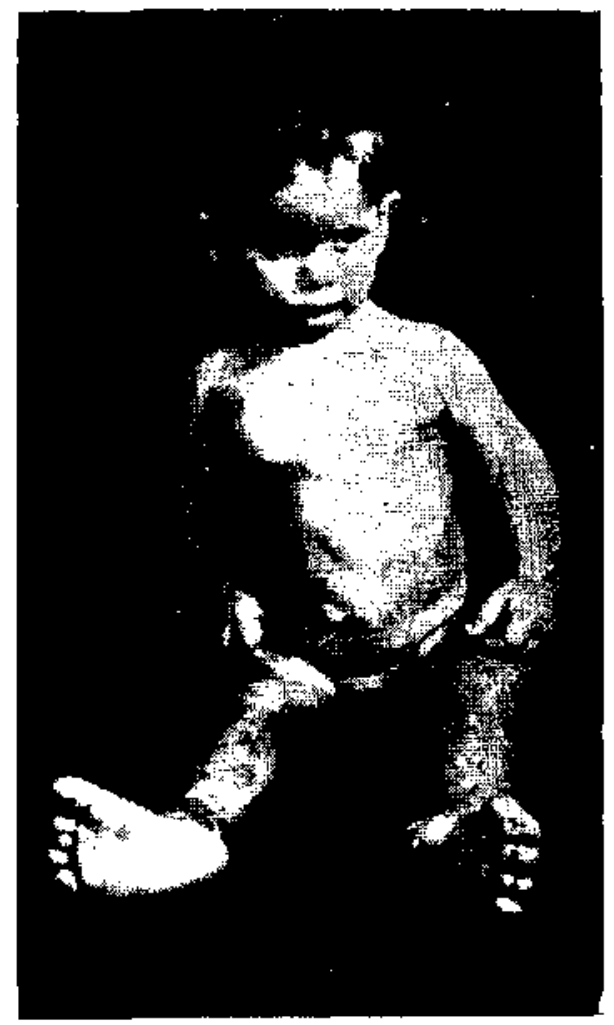

Fotografía No 1. - M. G. P. -- Ojs, 51/89418. Se aprecian angiomas planos. direminados, a nivel de las extremidades y tronco. Aumento de volumen dc las extremidades. especialmente $d$ ? las manos $y$ de los pies.

Sistema linfático: mieropoljadenias axilar, inguinal y submaxilar.

Cabeza: cráneo simétrico y osificado. Pelo corto. Región frontal algo prominente. Facies expresiva y rosada. ojos: conjuntivas rosadas, escleras azules, Reacciones pupilares normales. Boca: dentadura 11411; mucoza bucal húmeda y rosada. Faringe algo congestiva $y$ con secreción mucosa en la parte 
posterior. Lengua húmeda y limpia. Oidos: nada de especial. Cuello: normal.

Tórax: de consistencia algo blanda en la base, prominente en el $1 / 3$ inferior. Rosario costal discreto. Examen cardiopulmonar (-). Abdomen depresible, hernla umbilical. Hígado a $4 \mathrm{~cm}$. Bazo no se aprecla.
Se practica estudio histológlco, con biopsias cutáneas, practicadas a nivel del antebrazo izquierdo y de la región dorsal; en ellas se apreclan (fotos Nos. 3 y 4) dilataciones arteriolar y capilar como hecho fundamental. Existe tambiên ligera hiperqueratosis, infiltraclón mononuciear perivascular y edema en
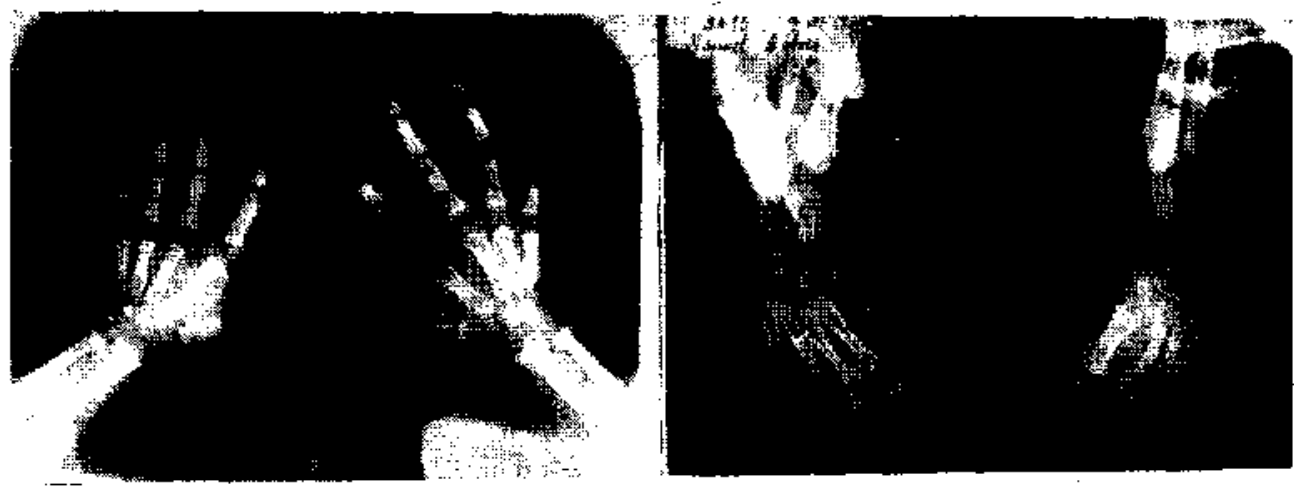

Foto $N^{1} 2$.

Radiografía de ambas manos $y$ pies: gigantismo $y$ aumento de la sombra de partes blandis de estos segmentos.

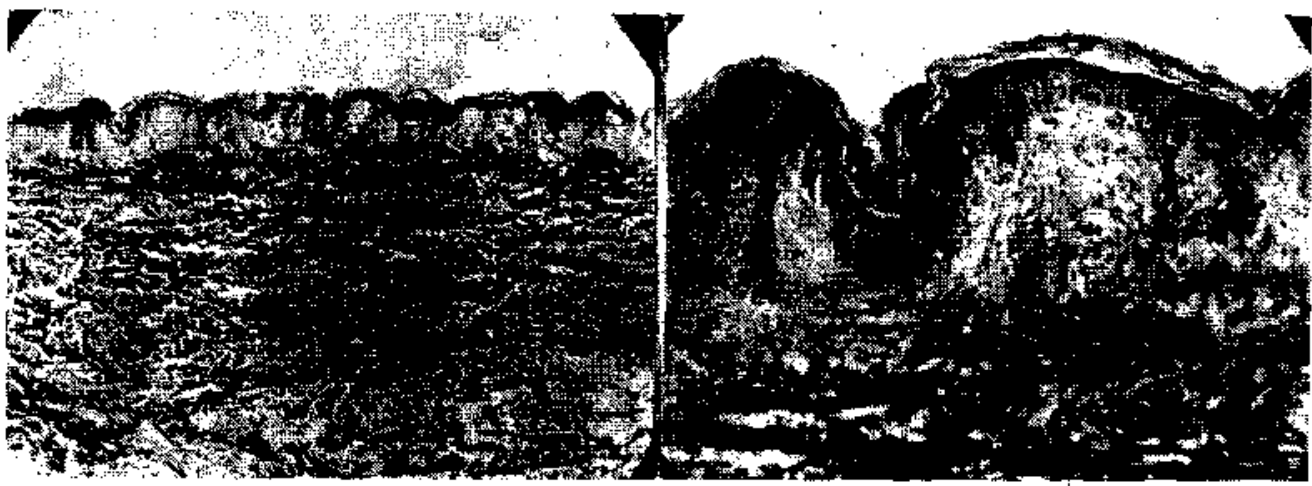

Fotografia Ne 3 .

Biopsia practicada a nivel de la piel del dorso. Obs. 51/89418. H. E. x 50. Edema y dilataciones vasculares, artariolares y capilares en el dermis.

Extremidades: gran htpotonia muscular, en especial de las inferiores. Se aprecia aumento de volumen notorlo de los ples y manos; los dedos estén algo incurvados, igual que los ortejos: es más evidente la deformación a derecha. Los pies están en varus. Hay pie plano bilateral.

Se plantea el diagnóstico de sindrome de Klifpel-Trenaunay, similar al cuadro clínico descrito por Parkes-Weber y que se caracteriza por la coexistencia de angiomas, várices e hipertrofia segmentaria.

\section{Fotografia N*4.}

Biopsia practicada a nivel de la piel del dorso. Obs, 51/89418. Corresponde a una sección de la microfotografia 3 , observada con anmento mayor (H, E. x 220). el dermis. Dres. R. Barahona y J. Esplnoza)

Exámenes complementarios: Radiografias de manos y pies: $N^{2} 3615$ (foto $N^{*} 2$ ).

"Gigantismo de ambas manos y pies. Allmento de sombras de las partes blandas de estos segmentos". (Dr. J. Hasbun).

Fosfemias: $4.09 \mathrm{mg} \%$

Fondo de ojo: normal.

Radioscopia de tórax: sombra alargada y nitida en el tercio medio derecho, en la region para-hiliar. 
Reacción de Kahn: negativa.

Exámenes de orlna $\mathrm{y}$ deposiciones: normales.

Hemograma: eritrocitos: $3.860,000 ; \quad \mathbf{H b}$ : $73.9 \%$; leucocitos: 5,300; Seg.: 38; Ltnf.: 50; EOS.: 8; Mono.: 4. Plaquetas, neutrólilos y linfocitos: normales.

\section{Comentario.}

En la observación relatada existió la tríada sintomática característica del sindrome de Klippel-Trenaunay, a saber: várices, angiomas e hipertrofia de algunos segmentos de las extremidades. Los angiomas se presentaron como manchas rosadas diseminadas en forma profusa $\mathrm{y}$ respetando únicamente la cara. Los trayectos varicosos fueron escasos y de ellos el de mayor magnitud correspondió a una vena superficial francamente dilatada a nivel de una amplia área del dorso. La hipertrofia regional se observó en los segmentos distales de las cuatro extremidades, sobresaliendo en forma muy llamativa el aumento considerable de volumen que adquírieron la mano y el pie del lado derecho, a este nivel como también en otras regiones, especialmente en la cara anteriox del tórax existia sensación especial, de verdadero cojimete, que traducía la hipertrofia tisural del subcutáneo. El estudio radiográfico en los segmentos distales de las extremidades demostró mayor longitud de algunos huesos, en relación a los correspondientes del lado opuesto, lo que revelaría la participación ósea en el aumento de volumen local. Los hallazgos histopatológicos más resaltantes en biopsias practicadas en la región dorsal, correspondieron a dilataciones vasculares y edema del dermis. Por oposición de los familiares fué imposible practicar en nuestro paciente un estudio venográfico, que podría haber conducido a la comprobación de la hipótesis patogénica de Servelle $y$ haber planteado la posibilidad de una intervención quirúrgica correctora.

\section{Sumario.}

Se describen las características del sindrome de Klippel-Trenaunay (coexistencia de angiomas, várices e hipertrofia regional) y se señala la similitud de este cuadro clínico, con la hemangiectasia hipertrófica de Parkes Weber.

Se expone la información bibliográfica de la afección y se insiste en la utilidad de la venografía, que puede comprobar anomalias vasculares, cuya corrección abriría posibilídades terapéuticas de orden quirúrgico.

Se relata la observación de un lactante de 1 año y 6 meses de edad, que presentó angiomas planos, várices e hipertrofia de las extremidades de predominio distal, con las peculiaridades senaladas en el síndrome de Klippel-Trenaunay.

\section{Summary.}

A case of an 18 months old infants with flat angiomas, varices, and distal hypertrophy of the extremities, with the peculiarities proper to Klippel-Trenaunay's syndrome, is reported.

\section{Bibliografía.}

AIMES, A. - Maladies et syndromes rares ou peu connus. Masson et Cle. Ed. Paris. 1850 .

CASATBBON y CHAIT (1930). - Citados por Cienfuegos, E. (1931).

CIENTUEGHOS, E. y MATTAR, A. - Hemanglectasia hipertrófica de Parkes Weber. Un caso de afección simétrica de ambas extremidades inferiores. Rev, Chilena de Pediat. 2: 121, 1931 .

PAUTRIER, L. M. y ULIMO, A. - Hemanglectasle hypertrophique de Parkes Weber. Bull. Dermat, et de $5 y p h$, pág. 981. 1828.

SERVELLW, M. - La veinographie va-t-elle nous permettre de demembrer le syndrome de Klippel et Trenaunay et l'hemangiectasie hipertrophique de Parkes Weber? Presse Méd. 26: 3is, 1945 .

TOURAINE, A. - Hemangiectasie hypertrophique de Parkes Weber. Encyclopedie Medico-chirurgical. Dermatologie. 1936. 